\title{
Radio constraints on dark matter annihilation in Canes Venatici I with LOFAR*
}

\author{
Martin Vollmann ${ }^{1}, \dagger$ Volker Heesen $^{2}$, Timothy Shimwell ${ }^{3}$, Martin J. Hardcastle ${ }^{4}$, \\ Marcus Brüggen ${ }^{2}$, Günter Sigl ${ }^{5}$ and Huub Röttgering ${ }^{6}$ \\ ${ }^{1}$ Physik Department T31. James-Franck-Straße 1, Technische Universität München, D-85748 Garching, Germany \\ ${ }^{2}$ Hamburger Sternwarte, Gojenbergsweg 112, D-21029 Hamburg, Germany \\ ${ }^{3}$ ASTRON, The Netherlands Institute for Radio Astronomy, Postbus 2, 7990 AA Dwingeloo, The Netherlands \\ ${ }^{4}$ Centre for Astrophysics Research, School of Physics, Astronomy and Mathematics, University of Hertfordshire, \\ College Lane, Hatfield AL10 9AB, UK \\ ${ }^{5}$ II. Institut für theoretische Physik, Universität Hamburg, Luruper Chaussee 149, D-22761 Hamburg, Germany \\ ${ }^{6}$ Leiden Observatory, Leiden University, PO Box 9513, NL-2300 RA Leiden, The Netherlands
}

Accepted <year month day>. Received <year month day >; in original form <year month day $>$.

\begin{abstract}
Dwarf galaxies are dark matter-dominated and therefore promising targets for the search for weakly interacting massive particles (WIMPs), which are well-known candidates for dark matter. Annihilation of WIMPs produce ultra-relativistic cosmic-ray electrons and positrons that emit synchrotron radiation in the presence of magnetic fields. For typical magnetic field strengths (few $\mu \mathrm{G}$ ) and $O(\mathrm{GeV}-\mathrm{TeV}$ ) WIMP masses (and thus typical electron energies of the same order) this emission peaks at hundreds of $\mathrm{MHz}$. Here, we use the non-detection of $150-\mathrm{MHz}$ radio continuum emission from the dwarf spheroidal galaxy 'Canes Venatici I' with the LOw-Frequency ARray (LOFAR) to derive constraints on the annihilation cross section of WIMPs into primary electron-positron and other fundamental particle-antiparticle pairs. In this first-of-itskind LOFAR study, we obtain new constraints on annihilating WIMP dark matter $(\mathrm{DM})$. Using conservative estimates for the magnetic field strengths and diffusion coefficients, we obtain limits that are comparable with those by the Fermi Large Area Telescope (Fermi-LAT) using gamma-ray observations. Assuming s-wave annihilation and WIMPs making up $100 \%$ of the DM density, our limits exclude several thermal WIMP realisations in the [2, 20]-GeV mass range. A more ambitious multi-wavelength and multi-target LOFAR study could improve these limits by a few orders of magnitude.
\end{abstract}

Key words: astroparticle physics - dark matter - galaxies:dwarf

\section{INTRODUCTION}

The $\Lambda$ CDM model provides a very successful description of most cosmological observations (see Planck Collaboration et al. 2016, for an overview). Perhaps most important, cold dark matter can explain the cosmic mass distribution as a result of its gravitational effects. Weakly interacting massive particles (WIMPs) are very appealing candidates for dark matter (DM) and by far the most scrutinised. These particles are required to have masses in the $\mathrm{GeV}$ to $\mathrm{TeV}$ range and interaction rates that can be accommodated with extensions of the standard model (SM) of particle physics in a rather straightforward manner. The thermal freeze-out of WIMPs

* Preprint numbers: TUM-1225/19

$\dagger$ E-mail: martin.vollmann@tum.de

(C) 2019 The Authors of mass $m_{\chi}$ occurs at a temperature $T=T_{f} \simeq m_{\chi} / 20$ which results in a relic mass density relative to the critical density today of Jungman et al. (1996)

$\Omega_{\chi} h^{2} \sim \frac{3 \times 10^{-27} \mathrm{~cm}^{3} / \mathrm{s}}{\left\langle\sigma_{\chi \chi} v\right\rangle}$,

where $\left\langle\sigma_{\chi} \bar{\chi} v\right\rangle$ is the total annihilation cross-section multiplied with the relative velocity averaged over a thermal distribution. Since $\Omega_{\chi}$, i. e. the density parameter of DM in form of WIMPs (henceforth denoted with the greek letter

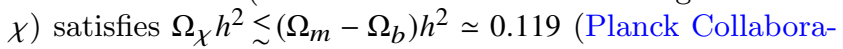
tion et al. 2016), eq. (1) puts a lower limit on the annihilation cross-section at the epoch of decoupling,

$\left\langle\sigma_{\chi \bar{\chi}} v\right\rangle \gtrsim\left\langle\sigma_{\mathrm{th}} v\right\rangle \simeq 3 \times 10^{-26} \mathrm{~cm}^{3} / \mathrm{s}$, 
where $\left\langle\sigma_{\mathrm{th}} v\right\rangle$ is known as thermal relic cross-section. The fact that it is of order an electroweak cross-section is referred to as the 'WIMP miracle' (Jungman et al. 1996).

Unfortunately, direct searches of these particles by the means of dedicated direct-detection and collider experiments have yielded only negative results; similarly, no indirect detection of DM by means of astronomical observations has been confirmed. In turn, these experiments and observations have put stringent constraints on several attractive WIMP models (Arcadi et al. 2018; Roszkowski et al. 2018).

Obviously, the discovery potential of any given DM experiment highly depends on the microscopic properties of the DM model. Diversified detection strategies such as the exploration of the low-frequency radio window for indirect detection of DM are thus essential. Annihilation of WIMP particles produces copious amounts of cosmic-ray (CR) electrons and positrons; they emit synchrotron radiation in the presence of magnetic fields. Due to synchrotron and inverseCompton scattering losses, CR electrons and positrons (CR $\left.e^{ \pm}\right)$are able to propagate only small distances without losing most of its energy (e. g. a few hundred parsecs for CR $e^{ \pm}$in the Milky Way Sigl (2017)). Thus, the otherwise undetectable excess of $\mathrm{CR} e^{ \pm}$due to DM annihilation can be probed with radio continuum observations.

Depending on the DM particle model, this synchrotron emission may be even the strongest signal in the context of multi-messenger astronomy. For example, in scotogenic and leptophilic DM models (Ma 2006; Fox \& Poppitz 2009), or in the context of super-symmetric sneutrino DM models, the DM particles couple to leptons rather than to quarks. These models have such properties that radio continuum observations in the hundreds of mega hertz range stand out as the most promising detection window, as long as the observed targets host strong enough magnetic fields.

Radio continuum observations were applied previously to the DM detection problem. To our knowledge Tyler (2002) was the first to make use of radio continuum observations of a dwarf galaxy. They obtained an upper limit for the 4.9-GHz flux density of the Draco dSph galaxy from observations with the Very Large Array. Similar recent studies are the ones of Regis et al. (2017); Leite et al. (2016); Marchegiani \& Colafrancesco (2016); Beck \& Colafrancesco (2016); Natarajan et al. (2015, 2013). Nevertheless, most of the indirect-detection searches with radio data have focused so far on other types of targets (mostly the Galactic Centre) (Bertone et al. 2001, 2002; Colafrancesco et al. 2006; Bertone et al. 2009; Fornengo et al. 2011, 2012a,b; Hooper et al. 2012; Carlson et al. 2013; Storm et al. 2013; Regis et al. 2014; Cirelli \& Taoso 2016; Storm et al. 2017; Lacroix et al. 2017; McDaniel et al. 2018); the same is true in the context of multi-messenger studies (Regis \& Ullio 2008).

In this Letter, we investigate the classical dwarf spheroidal (dSph) galaxy 'Canes Venatici I' (henceforth $\mathrm{CVnI}$ ). It is a satellite galaxy of the Milky Way at a distance of about $220 \mathrm{kpc}$ from the Sun at (J2000.0) R.A. $13^{\mathrm{h}} 28^{\mathrm{m}} 03^{\mathrm{s}} .5$ and Dec. $+33^{\circ} 33^{\prime} 21^{\prime \prime}$ (Zucker et al. 2006). It has a mass ${ }^{1}$ of $M=5.6 \times 10^{8} M_{\odot}$ and an azimuthally-averaged half-light radius of $r_{\star}=0.564 \mathrm{kpc}$ (Geringer-Sameth et al.

1 This is the mass that results from integrating the DM density (4) within a sphere with a radius of $2.03 \mathrm{kpc}$
2015). This dSph galaxy is among the 15 objects considered in the (6 yr) Fermi-LAT search for WIMPs study Ackermann et al. (2015).

Our theoretical predictions are based on a standard semi-analytical method that captures the annihilation physics, the diffusive CR propagation and the synchrotron radiation spectrum. We consider various scenarios for the diffusion coefficient and magnetic field strength. We do the same with the electron/positron production yields from the annihilation but for brevity only report here the results for exclusive (tree) annihilation into $e^{+} e^{-}$pairs. The corresponding results for the $\bar{b} b, \tau^{+} \tau^{-}, W^{+} W^{-}$, etc. are included in the appendix. This approach has become conventional in the literature as it facilitates the applicability of our results to a wider range of WIMP models.

Observations with the Low-Frequency Array (LOFAR) are used. LOFAR is an interferometric radio telescope operating at low frequencies (van Haarlem et al. 2013). We use maps from the preliminary second data release of the LOFAR Two-metre Sky Survey (LoTSS DR2; Shimwell et al. 2017, 2019), which is a deep 120-168 MHz imaging survey that will eventually cover the entire northern sky.

This Letter is organised as follows. In Section 2, we discuss the relevant phenomenology for WIMP searches with radio in dwarf galaxies; Section 3 presents the LOFAR observations; in Section 4 we show our constraints in the plane defined by the WIMP mass and annihilation cross-section into electron-positron pairs; we then conclude in Section 5.

\section{PREDICTIONS}

In order to obtain our theoretical predictions we follow the approach used in Leite et al. (2016), of which we give a brief summary in the following. Microscopic physics is captured by the annihilation cross-section into electrons and positrons, $\langle\sigma v\rangle\left(\chi \chi \rightarrow e^{ \pm \prime} \mathrm{s}+X\right)$, where the effects of the DM velocity distribution in the observed target are mostly negligible. Assuming that the DM is its own antiparticle, the rate at which the electrons and positrons are injected into the dSph galaxy's DM halo is given by:

$s\left(\boldsymbol{r}, E_{e^{ \pm}}\right)=\frac{1}{2 m_{\chi}^{2}} \rho^{2}(\boldsymbol{r}) \frac{\mathrm{d}\langle\sigma v\rangle}{\mathrm{d} E_{e^{ \pm}}}$.

The DM density $\rho(\boldsymbol{r})$ is assumed to be spherically symmetric with respect to the centre of the galaxy and it can be well described (Geringer-Sameth et al. 2015) by:

$$
\rho(r)=\frac{\rho_{s}}{\left(\frac{r}{r_{s}}\right)^{\gamma}\left[1+\left(\frac{r}{r_{s}}\right)^{\alpha}\right]^{\frac{\beta-\gamma}{\alpha}}},
$$

where $\rho_{s} c_{0}^{2}=0.5186 \mathrm{GeV} \mathrm{cm}^{-3}, r_{s}=3.56 \mathrm{kpc}, \alpha=1.8638$, $\beta=5.9969$, and $\gamma=0.6714$. The variable $r$ is the halo-centric radius and $c_{0}$ is the vacuum speed of light. This set of parameters is consistent with Ackermann et al. (2015) to ease comparison.

The quantity $\mathrm{d}\langle\sigma v\rangle / \mathrm{d} E_{e^{ \pm}}$is the velocity, angle and spin averaged DM annihilation cross-section into an electron (or positron) times the relative velocity per unit energy $E_{\boldsymbol{e}^{ \pm}}$. This quantity depends on the particle physics model. We can attain some model independence if we decompose the cross-sections as the linear superposition of products of hard 
$2 \rightarrow 2$ cross-sections $\left(\chi \chi \rightarrow e^{+} e^{-}, \chi \chi \rightarrow b \bar{b}\right.$, etc. $)$ times the differential $e^{ \pm}$yield from final-state particle cascades. The latter can be obtained from public Monte-Carlo software packages such as DarkSUSY (Gondolo et al. 2004) or PPPC (Cirelli et al. 2011). It follows that:

$\frac{\mathrm{d}\langle\sigma v\rangle}{\mathrm{d} E_{e^{ \pm}}}=\sum \operatorname{BR}\left(f^{+} f^{-}\right)\langle\sigma v\rangle_{f^{+} f^{-}} \frac{\mathrm{d} N_{f^{+} f^{-} \rightarrow e^{ \pm}+X}}{\mathrm{~d} E_{e^{ \pm}}}$,

where $f$ can be any particle of the SM.

Once the electrons and positrons are created by DM annihilation, their propagation can be well described by the diffusion-loss equation:

$\boldsymbol{\nabla} \cdot\left[D\left(\boldsymbol{r}, E_{e}\right) \boldsymbol{\nabla} n_{e^{ \pm}}\right]+b\left(\boldsymbol{r}, E_{e}\right) \frac{\partial n_{e^{ \pm}}}{\partial E}+s\left(\boldsymbol{r}, E_{e}\right)=0$,

where we assume spherical symmetry for simplicity. While most energy losses are due to the interaction of the electrons and positrons with the ambient electromagnetic field, the diffusion is due to the turbulent nature of the magnetic field. The relation between diffusion coefficient and magnetic field structure is complicated and model dependent. Approximately, in a turbulent field with an rms field strength $B$ and a power spectrum $\delta B^{2}(k)$ the diffusion coefficient for an electron is (Sigl 2017):

$$
\begin{aligned}
D(E) & \sim \frac{E}{3 e_{0} B} \frac{B^{2}}{\delta B^{2}\left(e_{0} B / E\right)} \\
& \sim 3 \times 10^{22}\left(\frac{E}{\mathrm{GeV}}\right)\left(\frac{\mu \mathrm{G}}{B}\right) \frac{B^{2}}{\delta B^{2}\left(e_{0} B / E\right)} \frac{\mathrm{cm}^{2}}{\mathrm{~s}},
\end{aligned}
$$

where $e_{0}$ is the elementary charge; note that $r_{L}=E /\left(e_{0} B\right)$ is the Larmor radius of an electron in a magnetic field $B$. The first factor in Equation (7) is referred to as the Bohm limit for diffusion. Because of the second factor, the real diffusion coefficient is always larger than the one obtained for the Bohm limit.

In the Milky Way, cosmic-ray abundance measurements, such as the boron to carbon ratio, give diffusion coefficients $D_{0} \equiv D(1 \mathrm{GeV})$ of the order of $10^{28} \mathrm{~cm}^{2} \mathrm{~s}^{-1}$ (e.g. Korsmeier \& Cuoco 2016), with similar values found in external galaxies for the CR $e^{-}$from spectral ageing (Heesen et al. 2019). These values are a factor of $\sim 10^{7}$ larger than for the Bohm limit. This is probably due to the small fractional magnetic field power $\delta B^{2}\left(10^{-6} \mathrm{pc}\right) / B^{2} \sim 10^{-7}$ at the Larmor radius $r_{L} \lesssim 10^{-6} \mathrm{pc}$ of a $\mathrm{GeV}$-electron. If the magnetic powerspectrum is a power-law with a slope of $n \sim-1$ at scales below the field coherence length $l_{c}$, one has $\delta B^{2}(k) \sim\left(k l_{c}\right)^{n}$. For $l_{c} \sim 10 \mathrm{pc}$, this results in the right order of magnitude. Given the large uncertainties of the magnetic field structure, we treat the diffusion coefficient and magnetic field strength as independent parameters for our purposes, as long as $D_{0} \sim 10^{28} \mathrm{~cm}^{2} \mathrm{~s}^{-1}$ and $B \sim 1 \mu \mathrm{G}$ hold within one to two orders of magnitude. In the following, we use for the parametrisation of the energy-dependency of the diffusion coefficient:

$D(E)=D_{0}\left(\frac{E}{\mathrm{GeV}}\right)^{\delta}$,

with $\delta=1+n$ in the model above. We will take $\delta=1 / 3$ which is supported by observations in the Milky Way (Korsmeier \& Cuoco 2016). As a first approximation, we further assume that the diffusion coefficient becomes infinity at a radius $r_{h}$ and that is homogeneous inside the sphere of the

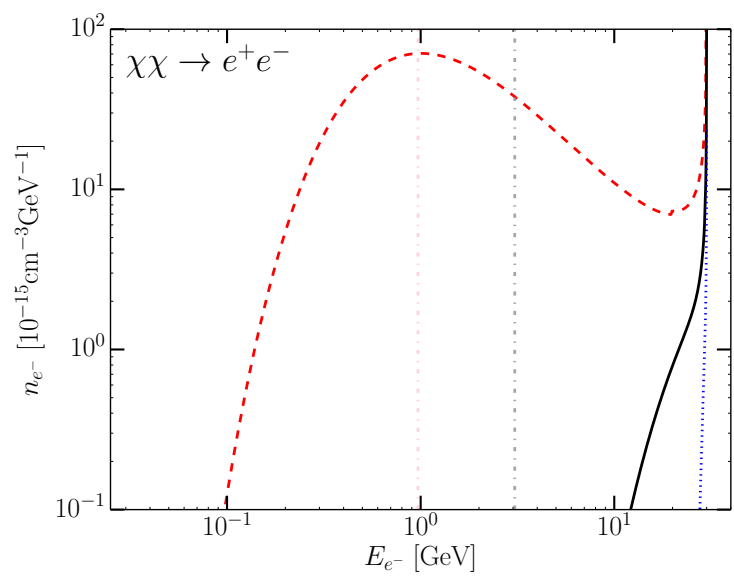

Figure 1. Electron/positron-density spectrum at the centre of the dSph galaxy resulting from the annihilation of $30-\mathrm{GeV}$ DM halo particles. We assume $B=10 \mu \mathrm{G}$ and $D_{0}=10^{26} \mathrm{~cm}^{2} \mathrm{~s}^{-1}$ (reddashed line); $B=1 \mu \mathrm{G}$ and $D_{0}=10^{27} \mathrm{~cm}^{2} \mathrm{~s}^{-1}$ (black-solid line); and $B=1 \mu \mathrm{G}$ and $D_{0}=10^{28} \mathrm{~cm}^{2} \mathrm{~s}^{-1}$ (blue-dotted line). Vertical lines mark the electron energy $E_{c}$ at which $v_{c}=150 \mathrm{MHz}$ for the two magnetic field strengths considered. See text for details.

same radius, an assumption that has become standard in the literature (Colafrancesco et al. (2007, 2006); McDaniel et al. (2017)). Then by adopting a semi-analytical approach it is possible to solve the diffusion-loss equation in terms of Green's functions (Vollmann 2019).

The resulting $\mathrm{CR} e^{ \pm}$distribution is shown in Fig. 1. It is evident that $\mathrm{CR} e^{ \pm}$originating from DM annihilation present rather distinctive features. The distribution becomes infinite at the mass of the DM particles, which is a consequence of the monochromatic energy distribution of the emitted electron-positron pairs per annihilation. The distribution features a low-energy cut-off at some specific $e^{ \pm}$ energy, which strongly depends on the diffusion coefficient and the magnetic field strength. Electrons and positrons at lower energies have diffused away from the dwarf galaxy.

Since the CR $e^{ \pm}$injection-rate density [Equation (3)] peaks at the centre of the dwarf galaxy and falls off towards the edges, we use $n_{e^{ \pm}}\left(r_{h}\right)=0$ at $r_{h}=1 \mathrm{kpc}$ as a boundary condition, adopting a radius of $2 r_{*}$. We verified that the (computationally favourable) boundary condition $n_{e^{ \pm}}\left(r_{h}\right)=0$ is compatible with the physical one: $D\left(r_{h}, E_{e}\right)\left(\partial n_{e^{ \pm}} / \partial r\right)\left(r_{h}\right)=c_{0} n_{e^{ \pm}}\left(r_{h}\right)$ in all the cases considered.

The radio emissivity associated with this synchrotron radiation is:

$j_{v}(\boldsymbol{r})=\int \mathrm{d} E_{e^{-}} 2 n_{e^{-}}\left(\mathbf{r}, E_{e}\right) P_{v}\left(E_{e^{-}}, B\right)$

where $P_{v}\left(E_{e^{-}}, B\right)$ is the pitch-angle averaged emitted power of a single electron in the presence of a magnetic field with rms strength $B$. The factor of 2 accounts for the fact that for CP-invariant models for DM as many positrons as electrons are produced in every annihilation. Then $P_{v}$ can be conveniently written as Leite et al. (2016):

$P_{\nu}\left(E_{e}\right)=\frac{9 \sqrt{3}}{8 \pi} \frac{b_{\mathrm{synch}}\left(E_{e}, B\right)}{v_{c}\left(E_{e}, B\right)} F\left(\frac{v}{v_{c}\left(E_{e}, B\right)}\right)$,

where $b_{\text {synch }}$ is the synchrotron energy-loss rate and Ghis- 


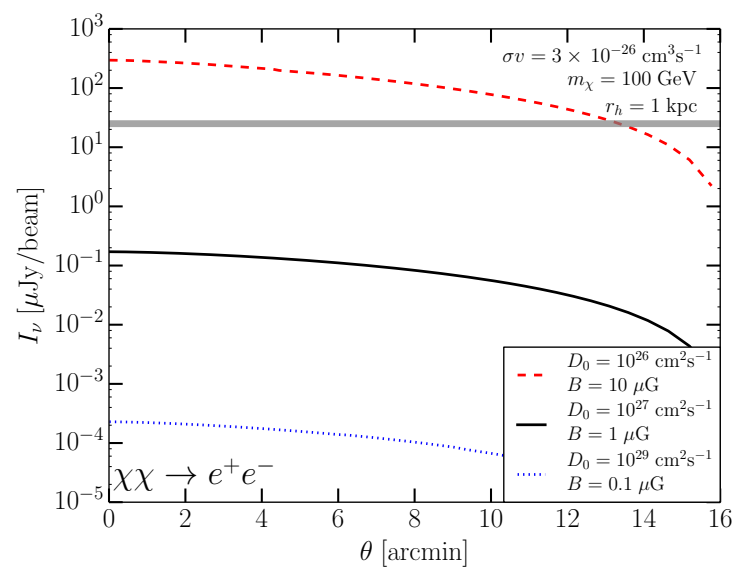

Figure 2. Predicted radial profile of the $150-\mathrm{MHz}$ radio continuum intensity for various combinations of diffusion coefficients $D_{0}$ and rms magnetic field strengths $B$. We assume a specific WIMP mass and annihilation cross-section into $e^{+} e^{-}$as indicated. This scenario corresponds to the electron/positron distribution as shown in Fig. 1.

ellini et al. (1988):

$F(x)=6 x^{2}\left[K_{4 / 3}(x) K_{1 / 3}(x)-\frac{3}{5}\left(K_{4 / 3}^{2}(x)-K_{1 / 3}^{2}(x)\right)\right]$,

and $v_{c}=3 e_{0} B E^{2} /\left(4 \pi m_{e}^{2}\right)$ is the critical frequency of the synchrotron radiation spectrum. In Fig. 1, we indicate the characteristic energy $E_{c}$ that results from inverting this equation and plugging in the observation frequency of $v_{c}=150 \mathrm{MHz}$. Electrons with energies smaller than this do not significantly emit synchrotron radiation at the observation frequency. The predicted radio continuum intensity is then:

$I_{v}=\int_{\operatorname{LoS}} j_{v}[\boldsymbol{r}(l)] \mathrm{d} l$,

which is the line-of-sight (LoS) integral of Equation (9).

Figure 2 shows the predicted $150-\mathrm{MHz}$ radio continuum intensity profile as a function of projected radius expressed by the apparent angle $\theta$; we assume $100-\mathrm{GeV}$ DM particles that annihilate into electron-positron pairs with the 'thermal' cross section. Three different scenarios are considered: (1) the optimistic scenario, where the magnetic field is strong $(10 \mu \mathrm{G})$ and highly turbulent (the diffusion constant is small $D=10^{26} \mathrm{~cm}^{2} \mathrm{~s}^{-1}$ for $\left.E_{e^{ \pm}}=1 \mathrm{GeV}\right) ;(2)$ the conservative scenario $\left(B=1 \mu \mathrm{G}, D_{0}=10^{27} \mathrm{~cm}^{2} \mathrm{~s}^{-1}\right)$; and (3) the pessimistic scenario, where energy losses can be neglected with $B=0.1 \mu \mathrm{G}$ and $D_{0}=10^{29} \mathrm{~cm}^{2} \mathrm{~s}^{-1}$. In scenario (3), neglecting energy losses is justified since the diffusion length within $\mathrm{CR} e^{ \pm}$lifetime exceeds the system size.

Notice that the optimistic case assumes a magnetic field that is much too large for a presumably quiet, nonstarforming dSph galaxy such as CVnI. We include it for completeness because it resembles most closely the limit in which the time-scale of diffusion is much larger than the corresponding $\mathrm{CR} e^{ \pm}$lifetime; the result serves also as a verification of the correctness of our predictions. Likewise, as a consequence of the strong dependence of our predictions on the diffusion coefficient and the magnetic field strength, our results are uncertain by several orders of magnitude.

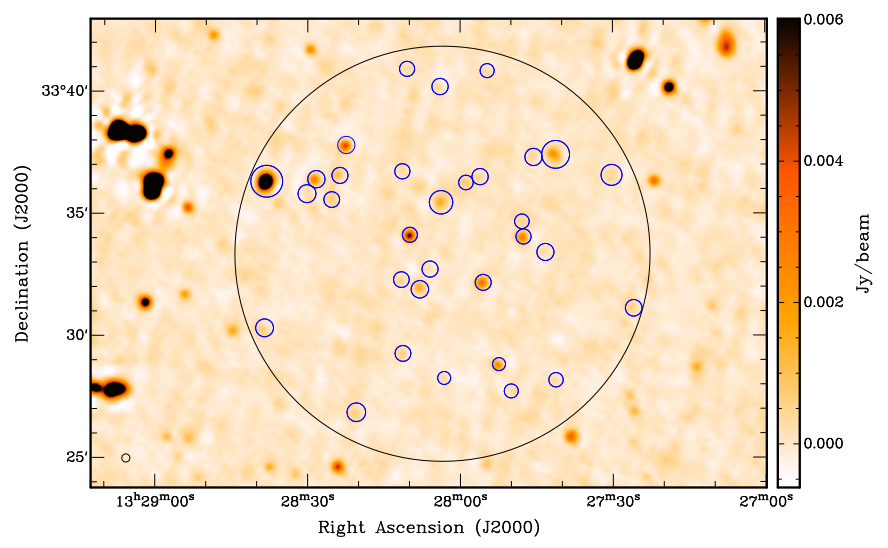

Figure 3. LoTSS $150-\mathrm{MHz}$ map of the region around $\mathrm{CVnI}$ at 20 arcsec FWHM angular resolution. We show the intensity at linear stretch between -0.6 and 6 mJybeam $^{-1}$. The large black circle indicates the area in which we integrated the intensity to measure the flux density, and the 32 small blue circles indicate the sources that we subtracted. The small black circle in the bottomleft corner shows the synthesized beam.

\section{LOFAR OBSERVATIONS}

We use a preliminary LoTSS DR2 150-MHz map at an angular resolution of 20 arcsec (full-width at half-maximum, FWHM) presented in Fig. 3. The optical radius of CVnI is 8.5 arcmin, which corresponds to 540 pc. We used a lower $(u, v)$-cut of $160 \lambda$, so that we are sensitive to emission on angular scales of up to approximately 21 arcmin; this is well above the size of the galaxy. A number of unresolved point-like sources can be seen, 32 of which are located within the 8.5 -arcmin radius. We detect no diffuse emission within this radius, which would be the expected morphology for DM-generated radio continuum emission; hence, we assume point-like sources to be unrelated to the galaxy. The correct way to ascertain the non-existence of diffuse emission is to integrate the intensity and check whether it is consistent with zero. For this we need to take rms map noise into account, which is $\sigma_{150 \mathrm{MHz}}=130 \mu \mathrm{Jy}$ beam $^{-1}$. Within a radius of 8.5 arcmin the flux density at $150 \mathrm{MHz}$ is $S_{150 \mathrm{MHz}}=(-3.6 \pm 5.5) \mathrm{mJy}$ after subtraction of the point-like sources, which contribute $89.5 \mathrm{mJy}$ in total. The resulting residual flux density is consistent with zero.

In order to test the estimated uncertainty, we inserted fake Gaussian sources into the $(u, v)$ data and then imaged and deconvolved the data in the same way as the original data. We found that a 5 -mJy Gaussian source can be detected with a FWHM of 3.3 arcmin if it is located cospatial with the optical centre of the galaxy. Less extended sources can be easily detected. We expect the dark matter signal to be strongly peaked in the centre of the galaxy since the particle annihilation and in turn the radio continuum emissivity scales with the square of the particle density. A further concentration is expected because the lines of sight are longer in the centre due to the spherical distribution of the dark matter. Hence we are confident that we can rule out any dark matter signal in the radio continuum as long as the dark matter distribution is sufficiently concentrated. 


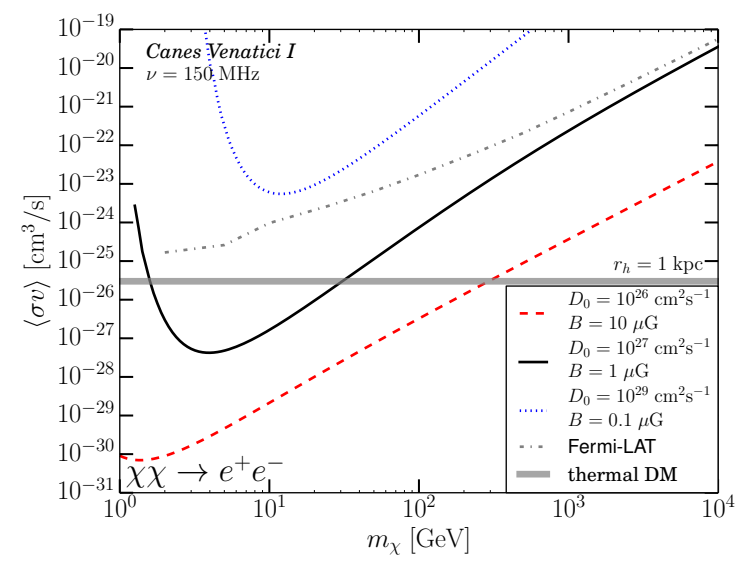

Figure 4. Resulting constraints on the WIMP annihilation crosssection into $e^{+} e^{-}$as a function of WIMP mass $m_{\chi}$, for various combinations of diffusion coefficients and magnetic field strengths. This scenario corresponds to the electron/positron distribution shown in Fig. 1 and the predicted radio continuum intensity profile in Fig. 2.

\section{WIMP CONSTRAINTS}

Bearing in mind that a thorough search for the DM signal discussed in Section 2 demands the employment of advanced data-analysis methods, we will content ourselves by estimating limits on $\langle\sigma v\rangle$ for several annihilation channels.

Concretely, we use the $2 \sigma$ limit on the maximum flux of a signal with a spherical tophat shape that can be extracted from a noisy image. This can be obtained analytically and is given by (Leite et al. (2016); Vollmann (2019)):

$I_{150 \mathrm{MHz}}^{\text {excluded at } 2 \sigma}=1.64 \frac{\sigma_{150 \mathrm{MHz}}}{\sqrt{N_{\text {beams }}}}$,

where $N_{\text {beams }}$ is the effective number of beams that are required to image the tophat signal (Vollmann (2019)). In practice, we estimated $N_{\text {beams }}$ as the ratio between the solid angle of a cone whose major and minor axes follow the $\mathrm{DM}$ distribution of $\mathrm{CVnI}$ and such that it contributes half of the total flux, to the solid angle corresponding to the Gaussian synthesized beam of the LOFAR map. In principle, $N_{\text {beams }}$ depends on the annihilation channel, DM particle mass, and diffusion coefficient. However, this dependence is weak and we find $\theta_{1 / 2}=4.75$ arcmin for scenario (1) and 4.10 arcmin, 4.09 arcmin for scenario (2) and (3), corresponding to $N_{\text {beams }}=478,355$ and 354 , respectively. Figure 4 shows the resulting constraints on the WIMP annihilation cross-section into $e^{+} e^{-}$as a function of mass resulting from comparing our radio continuum intensity predictions with the observational upper limit.

As a cautionary remark, the cross-section constraints obtained here are on present-time DM annihilation and they should thus not directly be compared with the thermal freeze-out annihilation cross-section $\left\langle\sigma_{\mathrm{th}} v\right\rangle$ that is relevant for the relic density. WIMP annihilation probed today is sensitive to relative velocities $v / c_{0} \sim 10^{-3}$. In contrast, thermal freeze-out is governed by the total annihilation crosssection at velocities $v / c_{0} \sim 0.3$. Therefore, a numerically much stronger constraint than $\left\langle\sigma_{\mathrm{th}} v\right\rangle$ can be reconciled with thermal freeze-out by either assuming an annihilation crosssection that decreases with $v$, such as in higher partial waves, or by assuming a branching ratio into electrons and positrons that is much smaller than unity; a combination of both is of course also possible.

\section{CONCLUSIONS}

Radio continuum observations of nearby dwarf galaxies offer the possibility to indirectly detect emission from dark matter, such as expected from the annihilation of WIMPs. For our educated guesses for the magnetic field strengths and the mass ballpark of the WIMP (few GeV to a few $\mathrm{TeVs}$ ), the synchrotron emission is expected to peak in the low-frequency radio continuum regime.

In this Letter, we have used a $150-\mathrm{MHz}$ map from the LOFAR Two-metre Sky Survey (LoTSS) to search for radio continuum emission from Canes Venatici I, a dSph satellite galaxy of the Milky Way. We do not detect any diffuse emission, allowing us to put constraints on the DM annihilation cross-sections into secondary electron and positron cascades for the generic DM models; we pay particular attention to primary hard electron-positron pairs from the $2 \rightarrow 2$ annihilation process. For WIMP masses of $2 \mathrm{GeV} \lesssim m_{\chi} \lesssim 20 \mathrm{GeV}$, the upper bounds on the primary $e^{+} e^{-}$process from our conservative scenario (2) are smaller than the total thermal relic cross-section. In the $[2 \mathrm{GeV}, 1 \mathrm{TeV}]$ energy interval, our limits are more stringent than the ones obtained with Fermi-LAT (see Fig. 4). A similar situation occurs when the electron and positrons from DM annihilation are produced by particle cascades from other leading scenarios of hard processes, such as $\chi \chi \rightarrow \tau^{+} \tau^{-}$, if stronger assumptions on the magnetic field strength and diffusion coefficients are made. This implies that either the total annihilation cross section today, which probes smaller relative velocities than relevant for freeze-out, has to be suppressed, for example due to a dominance of higher partial waves, or the branching ration into $e^{+} e^{-}$has to be small, or a combination thereof.

This proof-of-concept study is the first of its kind at the low frequencies probed by LOFAR. Since the predicted CR electron/positron distribution in Fig. 1 is fairly different from astrophysical spectra, the associated synchrotron signal benefits from distinctive features that can be explored in more ambitious multi-frequency and multi-object studies.

\section{ACKNOWLEDGMENTS}

MV and GS would like to thank the Max Planck Institute for Physics in Munich and particularly Prof. Raffelt for their hospitality and feedback while substantial parts of this work were completed. While completing this work, we became aware of two papers (Kar et al. (2019a,b)) where similar studies are performed but with different telescopes and targets. Their results are similar to those presented here.

This work is partly funded by the Deutsche Forschungsgemeinschaft under Germany's Excellence Strategy EXC 2121 "Quantum Universe" 390833306 and the Collaborative Research Center "Neutrinos and Dark Matter in Astroand Particle Physics" (SFB 1258). MJH acknowledges support from the UK Science and Technology Facilities Council (grant ST/R000905/1). This research has made use of the 
University of Hertfordshire high-performance computing facility (https://uhhpc.herts.ac.uk/) and the LOFAR-UK compute facility, located at the University of Hertfordshire and supported by STFC [ST/P000096/1].

LOFAR, the Low Frequency Array, designed and constructed by ASTRON, has facilities in several countries, which are owned by various parties (each with their own funding sources), and are collectively operated by the International LOFAR Telescope (ILT) foundation under a joint scientific policy. The ILT resources have benefited from the following recent major funding sources: CNRS-INSU, Observatoire de Paris and Université d'Orléans, France; BMBF, MIWF-NRW, MPG, Germany; Science Foundation Ireland (SFI), Department of Business, Enterprise and Innovation (DBEI), Ireland; NWO, The Netherlands; the Science and Technology Facilities Council, UK; Ministry of Science and Higher Education, Poland.

\section{REFERENCES}

Ackermann M., et al., 2015, Phys. Rev. Lett., 115, 231301

Arcadi G., Dutra M., Ghosh P., Lindner M., Mambrini Y., Pierre M., Profumo S., Queiroz F. S., 2018, Eur. Phys. J., C78, 203

Beck G., Colafrancesco S., 2016, JCAP, 1605, 013

Bertone G., Sigl G., Silk J., 2001, MNRAS, 326, 799

Bertone G., Sigl G., Silk J., 2002, MNRAS, 337, 98

Bertone G., Cirelli M., Strumia A., Taoso M., 2009, JCAP, 0903, 009

Carlson E., Hooper D., Linden T., Profumo S., 2013, JCAP, 1307, 026

Cirelli M., Taoso M., 2016, JCAP, 1607, 041

Cirelli M., et al., 2011, JCAP, 1103, 051

Colafrancesco S., Profumo S., Ullio P., 2006, Astron. Astrophys., 455,21

Colafrancesco S., Profumo S., Ullio P., 2007, Phys. Rev., D75, 023513

Fornengo N., Lineros R., Regis M., Taoso M., 2011, Phys. Rev. Lett., 107, 271302

Fornengo N., Lineros R. A., Regis M., Taoso M., 2012a, JCAP, 1201, 005

Fornengo N., Lineros R., Regis M., Taoso M., 2012b, JCAP, 1203, 033

Fox P. J., Poppitz E., 2009, Phys. Rev., D79, 083528

Geringer-Sameth A., Koushiappas S. M., Walker M., 2015, ApJ, 801, 74

Ghisellini G., Guilbert P. W., Svensson R., 1988, ApJ, 334, L5

Gondolo P., Edsjo J., Ullio P., Bergstrom L., Schelke M., Baltz E. A., 2004, JCAP, 0407, 008

Heesen V., et al., 2019, A\&A, 622, A8

Hooper D., Belikov A. V., Jeltema T. E., Linden T., Profumo S., Slatyer T. R., 2012, Phys. Rev., D86, 103003

Jungman G., Kamionkowski M., Griest K., 1996, Phys. Rept., 267,195

Kar A., Mitra S., Mukhopadhyaya B., Choudhury T. R., Tingay S., 2019b

Kar A., Mitra S., Mukhopadhyaya B., Choudhury T. R., 2019a

Korsmeier M., Cuoco A., 2016, Phys. Rev., D94, 123019

Lacroix T., Karami M., Broderick A. E., Silk J., Boehm C., 2017, Phys. Rev., D96, 063008

Leite N., Reuben R., Sigl G., Tytgat M. H. G., Vollmann M., 2016, JCAP, 1611, 021

Ma E., 2006, Phys. Rev., D73, 077301

Marchegiani P., Colafrancesco S., 2016, JCAP, 1611, 033

McDaniel A., Jeltema T., Profumo S., Storm E., 2017, JCAP, 1709,027
McDaniel A., Jeltema T., Profumo S., 2018, Phys. Rev., D97, 103021

Natarajan A., Peterson J. B., Voytek T. C., Spekkens K., Mason B., Aguirre J., Willman B., 2013, Phys. Rev., D88, 083535

Natarajan A., Aguirre J. E., Spekkens K., Mason B. S., 2015

Planck Collaboration et al., 2016, A\&A, 594, A13

Regis M., Ullio P., 2008, Phys. Rev., D78, 043505

Regis M., Colafrancesco S., Profumo S., de Blok W. J. G., Massardi M., Richter L., 2014, JCAP, 1410, 016

Regis M., Richter L., Colafrancesco S., 2017, JCAP, 1707, 025

Roszkowski L., Sessolo E. M., Trojanowski S., 2018, Rept. Prog. Phys., 81, 066201

Shimwell T. W., et al., 2017, A\&A, 598, A104

Shimwell T. W., et al., 2019, A\&A, 622, A1

Sigl G., 2017, Astroparticle Physics: Theory and Phenomenology. Atlantis Studies in Astroparticle Physics and Cosmology Vol. 1, Atlantis Press, doi:10.2991/978-94-6239-243-4

Storm E., Jeltema T. E., Profumo S., Rudnick L., 2013, Astrophys. J., 768, 106

Storm E., Jeltema T. E., Splettstoesser M., Profumo S., 2017, Astrophys. J., 839, 33

Tyler C., 2002, Phys. Rev. D, 66, 023509

Vollmann M., 2019, Under preparation

Zucker D. B., et al., 2006, ApJ, 643, L103

van Haarlem M. P., et al., 2013, A\&A, 556, A2

\section{APPENDIX A: FURTHER ANNIHILATION CHANNELS}

In this appendix we include our flux predictions for several annihilation channels (fig. A) and the limits that we obtain on the DM annihilation cross section into those channels. Specifically we considered $\tau^{+} \tau^{-}, W^{+} W^{-} \bar{q} q$ and $\bar{b} b$ where $\bar{q} q$ refers to any neutral pair of light quarks. 
Figure A1. Predicted brightness distributions for CVnI for different annihilation channels. Colour and dashing as in fig. 2
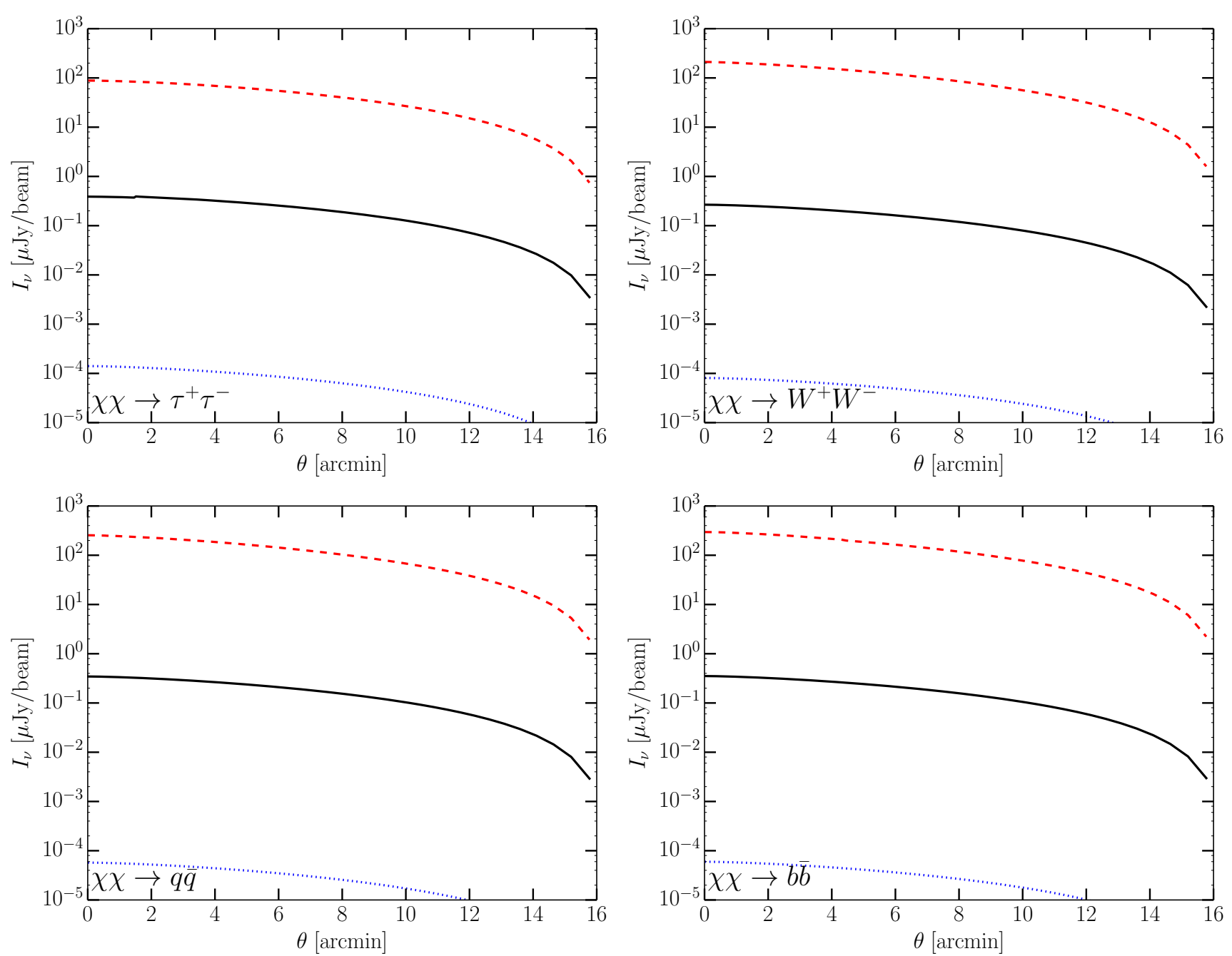
Figure A2. LOFAR CVnI limits on the cross section of annihilation of DM into several annihilation channels. Colour and dashing as in fig. 4
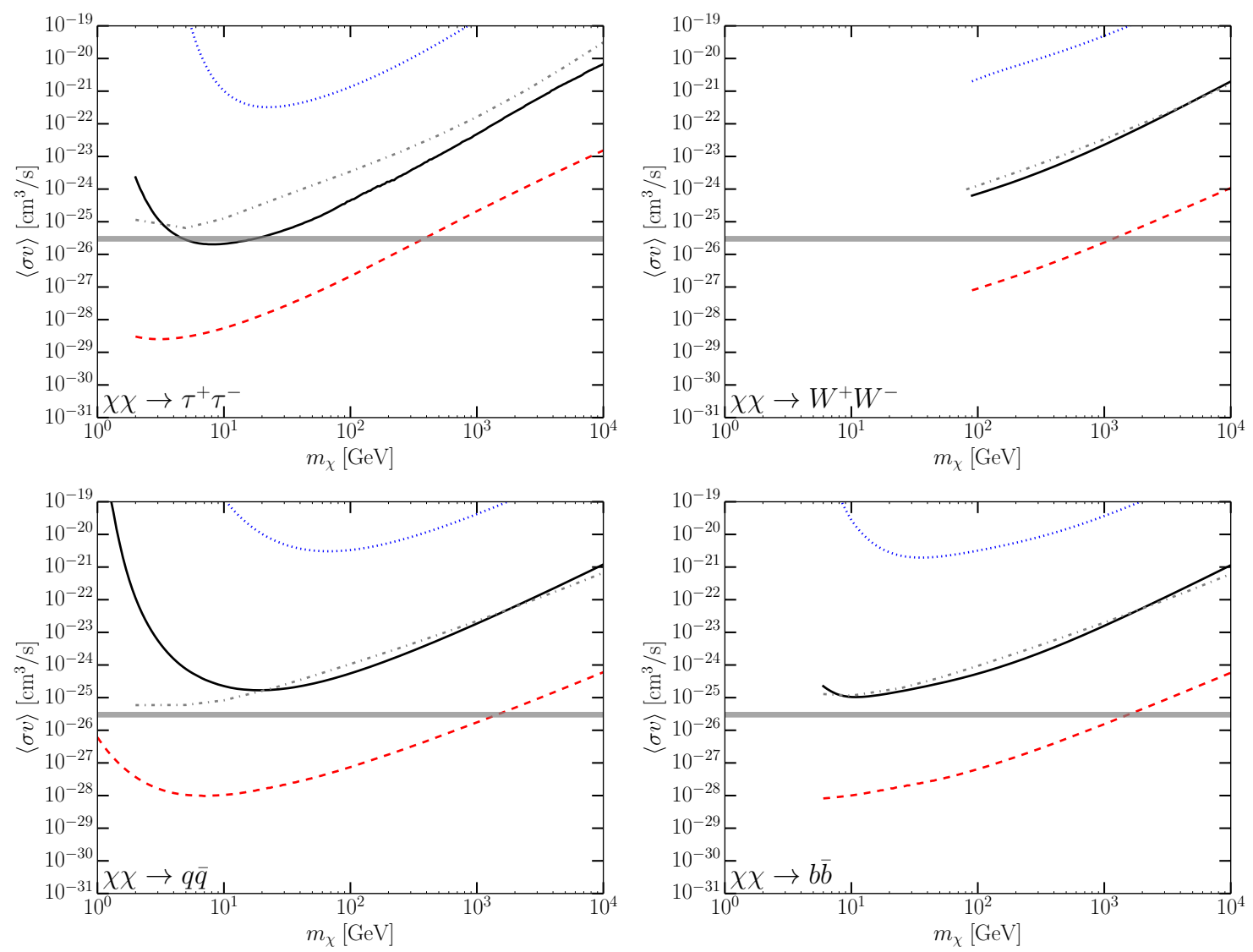